\title{
ON THE NUMBER OF REAL ROOTS OF A RANDOM ALGEBRAIC EQUATION
}

\author{
D. PRATIHARI, R. K. PANDA and B. P. PATTANAIK
}

(Received 20 June 1989; revised 23 February 1990)

Communicated by A. G. Pakes

\begin{abstract}
Let $N_{n}(w)$ be the number of real roots of the random algebraic equation $\sum_{\nu=0}^{n} a_{\nu} \xi_{\nu}(w) x^{\nu}=0$, where the $\xi_{\nu}(w)$ 's are independent, identically distributed random variables belonging to the domain of attraction of the normal law with mean zero and $P\left\{\xi_{\nu}(w) \neq 0\right\}>0$; also the $a_{\nu}$ 's are nonzero real numbers such that $\left(k_{n} / t_{n}\right)=O(\log n)$ where $k_{n}=\max _{0 \leq \nu \leq n}\left|a_{\nu}\right|$ and $t_{n}=\min _{0 \leq \nu \leq n}\left|a_{\nu}\right|$. It is shown that for any sequence of positive constants $\left(\varepsilon_{\mathrm{n}}, n \geq 0\right)$ satisfying $\varepsilon_{n} \rightarrow 0$ and $\varepsilon_{n}^{2} \log n \rightarrow \infty$ there is a positive constant $\mu$ so that

$$
\operatorname{Pr}\left\{\inf _{n>n_{0}} N_{n}(w) / \log n<\varepsilon_{n}\right\}<\mu\left(\varepsilon_{n_{0}} \log n_{0}\right)^{-1}
$$

for all $n_{0}$ sufficiently large.

1991 Mathematics subject classification (Amer. Math. Soc.): 60 B 99.

Keywords and phrases: Independent, identically distributed random variables, random algebraic equation, real roots, domain of attraction of the normal law, slowly varying function.
\end{abstract}

\section{Introduction}

Let $N_{n}(w)$ be the number of real roots of the algebraic equation

$$
f(x, w)=\sum_{\nu=0}^{n} \xi_{\nu}(w) x^{\nu}=0 ; \quad x \in \mathbf{R}
$$

where the $\xi_{\nu}(w)$ 's are independent, identically distributed real-valued random variables. Samal [7] has considered the general case when the $\xi_{\nu}(w)$ 's

(C) 1993 Australian Mathematical Society $0263-6115 / 93 \$ A 2.00+0.00$ 
are independent random variables identically distributed with expectation zero, the variance and the third absolute moment finite and nonzero. He has shown that $N_{n}(w)>\varepsilon_{n} \log n$ outside an exceptional set whose measure tends to zero as $n$ tends to infinity, where $\varepsilon_{n} \rightarrow 0$ but $\varepsilon_{n} \log n \rightarrow \infty$.

Mishra et al. [4] consider the equation

$$
f(x, w)=\sum_{\nu=0}^{n} a_{\nu} \xi_{\nu}(w) x^{\nu}=0
$$

in which the $\xi_{\nu}(w)$ 's are independent, identically distributed random variables belonging to the domain of attraction of the normal law with $P\left\{\xi_{\nu}(w) \neq 0\right\}>0$ and $a_{\nu}$ 's are nonzero real numbers such that

$$
k_{n}=\max _{0 \leq \nu \leq n}\left|a_{\nu}\right|, t_{n}=\min _{0 \leq \nu \leq n}\left|a_{\nu}\right|, k_{n} / t_{n}=O(\log n) .
$$

They show that when $n>n_{0}$,

$$
N_{n}(R, w)>(\mu \log n) / \log \left\{\frac{k_{n}}{t_{n}} \log n\right\}
$$

outside a set of measure at most

$$
\mu^{\prime} /\left\{\log \left(\frac{k_{n}}{t_{n}} \log n\right) \cdot(\log n)^{1-\varepsilon}\right\}
$$

for $0<\varepsilon<1$ and positive constants $\mu$ and $\mu^{\prime}$.

Mishra et al. [5] consider the polynomial equation (1.2) under the conditions (1.3) and prove that there exists a positive integer $n_{0}$ such that for $n>n_{0}$ and positive constants $C$ and $C^{\prime}$,

$$
N_{n}(R, w)>C\left\{\log n / \log \left(\frac{k_{n}}{t_{n}} \log \log n\right)\right\}^{1 / 2}
$$

outside a set of measure at most

$$
C^{\prime}\left\{\log \left(\frac{k_{n_{0}}}{t_{n_{0}}} \log \log n_{0}\right) / \log n_{0}\right\}^{(1-\varepsilon) / 2} ; \quad 0<\varepsilon<1 .
$$

The result (1.4) and (1.5) is of the form

$$
\operatorname{Pr}\left\{N_{n}(R, w) / \log n<\mu / \log \left(\frac{k_{n}}{t_{n}} \log n\right)\right\} \rightarrow 0,
$$

while the result contained in (1.6) and (1.7) is of the form:

$$
\operatorname{Pr}\left\{\inf _{n>n_{0}} N_{n}(R, w) / \log n<c /\left\{\log n \log \left(\frac{k_{n}}{t_{n}} \log \log n\right)\right\}^{1 / 2}\right\} \rightarrow 0 .
$$


The latter result is called the 'strong result' and may be referred to as the strong-version or 0 -version of the former.

Mishra et al. [6] solve the same problem, obtaining for $n>n_{0}$,

$$
N_{n}(R, w)>\varepsilon_{n} \log n
$$

outside an exceptional set of measure at most

$$
\mu /\left\{\varepsilon_{n} \log n+\left(k_{n} / t_{n}\right)^{\beta} \exp \left(-\mu^{\prime} \beta / \varepsilon_{n}\right)\right\},
$$

$(0<\beta<2-\varepsilon, 0<\varepsilon<2)$, provided that $\lim _{n \rightarrow \infty}\left(k_{n} / t_{n}\right)$ is finite.

Earlier Samal and Pratihari [10] had obtained the lower bound (1.8) with an exceptional set of measure smaller than (1.9) in case the $\xi_{\nu}(w)$ 's are independent and identically distributed random variables with common characteristic function $\exp \left(-C|t|^{\alpha}\right) ; C$ being a positive constant and $\alpha \geq 1$. Samal and Pratihari [8] have proved the 0-version of their theorems in [10] with refinement of their exceptional set and they have extended this result to the general case in [9] when the $\xi_{\nu}(w)$ 's are independent, identically distributed random variables with mean zero and the variance and the third absolute moment finite and nonzero. They have obtained the lower bound (1.8) outside an exceptional set of measure at most $\mu /\left(\varepsilon_{n_{0}} \log n_{0}\right)$ for $n>n_{0}, n_{0}$ being sufficiently large and $\mu$ a positive constant. It is apparent that Mishra, Nayak and Pattanayak are not aware of $[8,9,10]$.

In this paper our object is to prove the following theorem.

THEOREM. Let $N_{n}(w)$ be the number of real roots of the equation $f(x, w)$ $=\sum_{\nu=0}^{n} a_{\nu} \xi_{\nu}(w) x^{\nu}=0$ of degree $n$, where the coefficients $\xi_{\nu}(w)$ are independent, identically distributed random variables belonging to the domain of attraction of the normal law with mean zero and $\operatorname{Pr}\left\{\xi_{\nu}(w) \neq 0\right\}>0$. Let the $a_{\nu}$ 's be nonzero real numbers such that $k_{n} / t_{n}=O(\log n)$, where $k_{n}=\max _{0 \leq \nu \leq n}\left|a_{\nu}\right|, t_{n}=\min _{0 \leq \nu \leq n}\left|a_{\nu}\right|$. Then, for any sequence of positive constants $\left(\varepsilon_{n}, n \geq 0\right)$ satisfying $\varepsilon_{n} \rightarrow 0$ and $\varepsilon_{n}^{2} \log n \rightarrow \infty$, there is a positive constant $\mu$ so that

$$
\operatorname{Pr}\left\{\inf _{n>n_{0}} N_{n}(w) / \log n<\varepsilon_{n}\right\}<\mu\left(\varepsilon_{n_{0}} \log n_{0}\right)^{-1}
$$

for all $n_{0}$ sufficiently large.

This theorem gives the strong result of Mishra et al. [5] as a particular case. Choosing $\varepsilon_{n}=c /\left\{\log n \log \left(\frac{k_{n}}{t_{n}} \log \log n\right)\right\}^{1 / 2}$ in our theorem, their lower bound (1.6) is obtained. Moreover, for this choice of $\varepsilon_{n}$ our exceptional set becomes smaller than theirs (1.7). Of course, for such choice of $\varepsilon_{n}, \varepsilon_{n}^{2} \log n$ 
tends to zero instead of $\infty$, but $\left(\alpha \varepsilon_{n} \log n\right)^{2}$ tends to infinity. It will be seen in the sequel that $k$ appearing in (2.8) is a positive integer tending to infinity.

Throughout this paper [x] denotes the greatest integer not exceeding $x$, $V(\eta)$ the variance of the random variable $\eta$. We assume that all inequalities are satisfied for $n$ sufficiently large. Positive constants are denoted by $\mu$ 's.

\section{Proof of the theorem}

Since the $\xi_{\nu}(w)$ 's belong to the domain of attraction of the normal law, their characteristic function is given by (cf. Ibragimov and Linnik [3, page 91])

$$
\phi(t)=\exp \left\{-\frac{t^{2}}{2} h(t)\right\}
$$

where $h(t)$ is a slowly varying function as $t \rightarrow 0$ with the property that

$$
h(t)=\operatorname{Re} h(t)\{1+o(1)\} \text {. }
$$

Let

$$
h_{1}(t)= \begin{cases}\operatorname{Re} h(t) & \text { if } V\left(\xi_{\nu}\right)=\infty, \\ \sigma^{2} & \text { if } V\left(\xi_{\nu}\right)=\sigma^{2}<\infty,\end{cases}
$$

which is a slowly varying function in a neighbourhood of the origin. By (2.2), $h(t)=h_{1}(t)\{1+o(1)\}$ in both the cases as $t \rightarrow 0$.

2.1. Take absolute constants $A$ and $B$ such that $A>1$ and $0<B<1$. Choose

$$
\beta_{n}=\left(\frac{t_{n}}{k_{n}}\right) \exp \left\{C_{1} /\left(\varepsilon_{n}^{2} \log n\right)\right\}
$$

where $C_{1}$ is a constant to be chosen later. Let, for constants $d_{1}>1, e=$ $\exp (1)$,

$$
M_{n}=\left[\frac{d_{1}^{2}(\sqrt{2}+1)^{2}}{16} \beta_{n}^{2}\left(\frac{k_{n}}{t_{n}}\right)^{2} \cdot\left(\frac{A e}{B}\right)\right]+1
$$

so that

$$
\mu_{1}\left\{\left(k_{n} / t_{n}\right) \beta_{n}\right\}^{2} \leq M_{n} \leq \mu-2\left\{\left(k_{n} / t_{n}\right) \beta_{n}\right\}^{2}
$$

Let

$$
\phi(x)=x^{x}
$$

and $k$ be an integer determined by

$$
\phi(8 k+7) M_{n}^{8 k+7} \leq n<\phi(8 k+11) M_{n}^{8 k+11} .
$$


The first inequality of (2.7) gives

$$
(8 k+7)\left\{\log (8 k+7)+\log M_{n}\right\} \leq \log n
$$

and ultimately

$$
k \leq \mu^{\prime \prime}(\log n) / \log \left(\frac{k_{n}}{t_{n}} \beta_{n}\right) .
$$

The second inequality of $(2.7)$ gives

$$
\begin{aligned}
\log n & <(8 k+11)\left\{\log (8 k+11)+\log M_{n}\right\} \\
& <(8 k+11)^{2}+(8 k+11) \log M_{n} \\
& <\mu k^{2} \log M_{n},
\end{aligned}
$$

so that $k>\mu^{\prime}\left\{(\log n) / \log \left(k_{n} \beta_{n} / t_{n}\right)\right\}^{1 / 2}$. Thus, from (2.7) we have

$$
\frac{\mu_{1}}{\sqrt{C_{1}}} \varepsilon_{n} \log n \leq K \leq \frac{\mu_{2}}{C_{1}}\left(\varepsilon_{n} \log n\right)^{2} .
$$

We consider $f\left(x_{m}, w\right)=U_{m}(w)+R_{m}(w)$ at the points

$$
x_{m}=\left\{1-\frac{1}{\phi(4 m+1) M_{n}^{4 m}}\right\}^{1 / 2}
$$

for $m=[k / 2]+1,[k / 2]+2,[k / 2]+3, \ldots, k$, where

$$
U_{m}(w)=\sum_{1} a_{\nu} \xi_{\nu}(w) x_{m}^{\nu} \text { and } R_{m}(w)=\left(\sum_{2}+\sum_{3}\right) a_{\nu} \xi_{\nu}(w) x_{m}^{\nu},
$$

the index $\nu$ ranging from $\nu_{1}+1=\phi(4 m-1) M_{n}^{4 m-1}+1$ to $\nu_{2}=$ $\phi(4 m+3) M_{n}^{4 m+3}$ in $\Sigma_{1}$, from 0 to $\nu_{1}$ in $\sum_{2}$ and from $\nu_{2}+1$ to $n$ in $\Sigma_{3}$. So

$$
f\left(x_{2 m}, w\right)=U_{2 m}(w)+R_{2 m}(w) ; f\left(x_{2 m+1}, w\right)=U_{2 m+1}(w)+R_{2 m+1}(w)
$$

where $U_{2 m}(w)$ and $U_{2 m+1}(w)$ are independent. Let $V_{m}$ be given by the relation

$$
\frac{1}{V_{m}^{2}} \sum_{\nu=\nu_{1}+1}^{\nu_{2}} a_{\nu}^{2} x_{m}^{2 \nu} h_{1}\left(a_{\nu} x_{m}^{\nu} \theta / V_{m}\right)=1
$$

where $\theta$ is a small positive number to be chosen later. Ibragimov and Maslova [2] show that normalising constants such as $V_{m}$ exist under conditions of our theorem for $\theta$ sufficiently small. 
If $V\left(\xi_{\nu}(w)\right)=\sigma^{2}<\infty$, then

$$
\begin{aligned}
V_{m}^{2} & =\sigma^{2} \sum_{\nu=\nu_{1}+1}^{\nu_{2}} a_{\nu}^{2} x_{m}^{2 \nu} \geq \sigma^{2} t_{n}^{2} \sum_{\nu=\nu_{1}+1}^{\nu_{2}} x_{m}^{2 \nu} \\
& >\sigma^{2} t_{n}^{2} \phi(4 m+1) M_{n}^{4 m}(B / A e)
\end{aligned}
$$

or,

$$
\phi(4 m+1) M_{n}^{4 m}<(A e / B) \frac{V_{m}^{2}}{\sigma^{2} t_{n}^{2}} .
$$

If $V\left(\xi_{\nu}(w)\right)=\infty$, then we have by (2.2) $\lim _{t \rightarrow 0} h_{1}(t)=\infty$ so that we choose $\theta$ such that $h_{1}(t)>1$ for $|t|<\theta$. Hence, in this case we have

$$
\phi(4 m+1) M_{n}^{4 m}<(A e / B)\left(V_{m}^{2} / t_{n}^{2}\right) .
$$

2.2. We give here three lemmas to be used in the proof.

LEMMA 1. $\left|\sum_{2} a_{\nu} \xi_{\nu}(w) x_{m}^{\nu}\right|<\left(m \beta_{n}\right) W_{m}$ except for a set of measure at most $\mu /\left(m \beta_{n}\right)^{2-\varepsilon}$ for $\varepsilon>0$, where

$$
W_{m}^{2}=\sum_{2} a_{\nu}^{2} x_{m}^{2 \nu} h_{1}\left(a_{\nu} x_{m}^{\nu} \theta / W_{m}\right)
$$

LEMMA 2. $\left|\sum_{3} a_{\nu} \xi_{\nu}(w) x_{m}^{\nu}\right|<\left(m \beta_{n}\right) Z_{m}$ except for a set of measure at most $\mu /\left(m \beta_{n}\right)^{2-\varepsilon}$ for $\varepsilon>0$, where

$$
Z_{m}^{2}=\sum_{3} a_{\nu}^{2} x_{m}^{2 \nu} h_{1}\left(a_{\nu} x_{m}^{\nu} \theta / Z_{m}\right)
$$

These lemmas are proved in the same way as in Mishra et al. [4].

LEMMA 3. $\left|R_{m}(w)\right|<V_{m}$ except for a set of measure at most $\mu /\left(m \beta_{n}\right)^{2-\varepsilon}$ for $m=m_{0}, m_{0}+1, m_{0}+2, \ldots, k ; m_{0}=[k / 2]+1$.

Proof. (CASe I.) Let $V\left(\xi_{\nu}(w)\right)=\infty$ Then by Lemmas 1 and $2,\left|R_{m}\right|<$ $m \beta_{n}\left(W_{m}+Z_{m}\right)$ for any $m$, except for a set of measure at most $\mu /\left(m \beta_{n}\right)^{2-\varepsilon}$, $\varepsilon>0$. That is

$$
\begin{aligned}
\left|R_{m}\right| & <m \beta_{n}\left(\left\{\sum_{2} a_{\nu}^{2} x_{m}^{2 \nu} h_{1}\left(a_{\nu} x_{m}^{\nu} \theta / W_{m}\right)\right\}^{1 / 2}+\left\{\sum_{3} a_{\nu}^{2} x_{m}^{2 \nu} h_{1}\left(a_{\nu} x_{m}^{\nu} \theta / Z_{m}\right)\right\}^{1 / 2}\right) \\
& <m \beta_{n} k_{n} d\left(\left\{\sum_{2} x_{m}^{2 \nu}\right\}^{1 / 2}+\left\{\sum_{3} x_{m}^{2 \nu}\right\}^{1 / 2}\right)
\end{aligned}
$$


where $d=\max _{0 \leq \nu \leq \eta}\left(\left\{h_{1}\left(a_{\nu} x_{m}^{\nu} \theta / W_{m}\right)\right\}^{1 / 2},\left\{h_{1}\left(a_{\nu} x_{m}^{\nu} \theta / Z_{m}\right)\right\}^{1 / 2}\right)$.

Clearly $d>1$ since $\theta$ is small. Again, we can choose $\theta$ so that $h_{1}$ are bounded (cf. Mishra et al. [6, page 23]). Hence $d$ is bounded above. Let $d_{1}$ be a positive constant such that $d \leq d_{1}$. Then

$$
\left|R_{m}\right|<m \beta_{n} k_{n} d_{1}\left(\left\{\sum_{2} x_{m}^{2 \nu}\right\}^{1 / 2}+\left\{\sum_{3} x_{m}^{2 \nu}\right\}^{1 / 2}\right) .
$$

Again

$$
\begin{aligned}
\sum_{\nu=0}^{\phi(4 m-1) M_{n}^{4 m-1}} x_{m}^{2 \nu} & <\phi(4 m-1) M_{n}^{4 m-1}+1 \\
& <2 \phi(4 m-1) M_{n}^{4 m-1}<\frac{2 \phi(4 m+1) M_{n}^{4 m}}{16 m^{2} \cdot M_{n}}
\end{aligned}
$$

Also

$$
\begin{aligned}
\sum_{\nu=\phi(4 m+3) M_{n}^{4 m+3}+1}^{n} x_{m}^{2 \nu} & <\sum_{\nu=\phi(4 m+3) M_{n}^{4 m+3}+1}^{\infty} x_{m}^{2 \nu} \\
& =\frac{x^{2\left\{\phi(4 m+3) M_{n}^{4 m+3}+1\right\}}}{1-x_{m}^{2}} \\
& <\phi(4 m+1) M_{n}^{4 m}\left\{1-\frac{1}{\phi(4 m+1) M_{n}^{4 m}}\right\}^{\phi(4 m+3) M_{n}^{4 m+3}} .
\end{aligned}
$$

Now $\phi(4 m+3) M_{n}^{4 m+3}>\phi(4 m+1) M_{n}^{4 m}(4 m+1)^{2} M_{n}^{2}$ so that

$\sum_{3} x^{2 \nu}<\phi(4 m+1) M_{n}^{4 m} \exp \left\{-(4 m+1)^{2} M_{n}^{2}\right\}<\phi(4 m+1) M_{n}^{4 m} /\left(16 m^{2} M_{n}\right)$.

Therefore, using (2.16), (2.17) and (2.13) we get

$$
\left|R_{m}\right|<\frac{(\sqrt{2}+1)}{4} d_{1} \beta_{n} k_{n}\left\{\phi(4 m+1) M_{n}^{4 m}\right\}^{1 / 2} / M_{n}^{1 / 2}<V_{m} .
$$

(CASE II.) When $V\left(\xi_{\nu}(w)\right)=\sigma^{2}<\infty$, we have

$$
\begin{aligned}
\left|R_{m}\right| & <m \beta_{n} k_{n} \sigma\left\{\left(\sum_{2} x_{m}^{2 \nu}\right)^{1 / 2}+\left(\sum_{3} x_{m}^{2 \nu}\right)^{1 / 2}\right\} \\
& <\frac{(\sqrt{2}+1)}{4} \sigma \beta_{n} k_{n}\left\{\phi(4 m+1) M_{n}^{4 m}\right\}^{1 / 2} / M_{n}^{1 / 2}<V_{m} .
\end{aligned}
$$


Hence $\left|R_{m}\right|<V_{m}$ in both cases and for $m=m_{0}, m_{0}+1, \ldots, k$ except for a set of measure at most $\mu /\left(m \beta_{n}\right)^{2-\varepsilon}$.

2.3. We define events $E_{m}$ as the sets of $w$ for which $U_{2 m}(w)>V_{2 m}$ and $U_{2 m+1}(w)<-V_{2 m+1}$ and the events $F_{m}$ as the sets of $w$ for which $U_{2 m}(w)<-V_{2 m}$ and $U_{2 m+1}(w)>V_{2 m+1}$.

Let $S_{m}^{+}, S_{m}^{-}$be the sets of $w$ in which $U_{m}(w)>V_{m}$ and $U_{m}(w)<-V_{m}$ respectively. Hence $E_{m} \cup F_{m}=\left(S_{2 m}^{+} \cap S_{2 m+1}^{-}\right) \cup\left(S_{2 m}^{-} \cap S_{2 m+1}^{+}\right)$. Since the two sets within the braces on the right hand side are disjoint and since $U_{2 m}(w)$ and $U_{2 m+1}(w)$ are independent random variables, we have

$$
\begin{aligned}
P= & P\left(E_{m} \cup F_{m}\right)=P\left(S_{2 m}^{+}\right) P\left(S_{2 m+1}^{-}\right)+P\left(S_{2 m}^{-}\right) P\left(S_{2 m+1}^{+}\right) \\
= & P\left(U_{2 m}>V_{2 m}\right) P\left(U_{2 m+1}<-V_{2 m+1}\right) \\
& +P\left(U_{2 m}<-V_{2 m}\right) P\left(U_{2 m+1}>V_{2 m+1}\right)=\delta_{m} \text { (say). }
\end{aligned}
$$

Let $G_{m}(x)$ and $g_{m}(t)$ be respectively the distribution function and the characteristic function of $\left(U_{m} / V_{m}\right)$. Then

$$
g_{m}(t)=\exp \left\{-\frac{t^{2}}{2} \cdot \frac{1}{V_{m}^{2}} \sum_{\nu=\nu_{1}+1}^{\nu_{2}} a_{\nu}^{2} x_{m}^{2 \nu} h\left(a_{\nu} x_{m}^{\nu} t / V_{m}\right)\right\} .
$$

Let $F(x)=(1 / \sqrt{2 \pi}) \int_{-\infty}^{x} \exp \left(-u^{2} / 2\right) d u$. As in [6], as $m \rightarrow \infty, g_{m}(t) \rightarrow$ $\exp \left(-t^{2} / 2\right)$ in any bounded interval of $t$-values. Hence

$$
\sup _{x}\left|G_{m}(x)-F(x)\right|=o(1) .
$$

So $\left|G_{2 m}(-1)-f(-1)\right|<\varepsilon$ and $\left|G_{2 m+1}(-1)-F(-1)\right|<\varepsilon ; \varepsilon>0$. Thus, from (2.18), we get

$$
P=\delta_{m}>2\{F(-1)-\varepsilon\}\{1-F(1)-\varepsilon\}=\delta \text { (say). }
$$

Obviously $\delta_{m}>\delta>0$ for large values of $m$.

2.4. Let $\eta_{m}$ be a random variable such that it takes values 1 on $E_{m} \cup F_{m}$ and zero elsewhere. In other words,

$$
\eta_{m}=\left\{\begin{array}{l}
1 \text { with probability } \delta_{m} \\
0 \text { with probability } 1-\delta_{m}
\end{array}\right.
$$

The $\eta_{m}$ 's are thus random variables with $E\left(\eta_{m}\right)=\delta_{m}$ and $V\left(\eta_{m}\right)=\delta_{m}-$ $\delta_{m}^{2}<1$.

Let $\rho_{m}$ be defined as follows:

$$
\rho_{m}= \begin{cases}0 & \text { if }\left|R_{2 m}\right|<V_{2 m} \text { and }\left|R_{2 m+1}\right|<V_{2 m+1} \\ 1 & \text { elsewhere, }\end{cases}
$$

where $(2.10)$ holds. 
Let $\theta_{m}=\eta_{m}-\eta_{m} \rho_{m}$. Now $\theta_{m}=1$ only if $\eta_{m}=1$ and $\rho_{m}=0$, which implies the occurrence of one of the events:

(i) $U_{2 m}>V_{2 m},\left|R_{2 m}\right|<V_{2 m} ;\left|U_{2 m+1}\right|<-V_{2 m+1},\left|R_{2 m+1}\right|<V_{2 m+1}$.

(ii) $U_{2 m}<-V_{2 m},\left|R_{2 m}\right|<V_{2 m} U_{2 m+1}>V_{2 m+1},\left|R_{2 m+1}\right|<V_{2 m+1}$.

It is obvious that (i) implies $f\left(x_{2 m}\right)>0$ and $f\left(x_{2 m+1}\right)<0$ and (ii) implies that $f\left(x_{2 m}\right)<0$ and $f\left(x_{2 m+1}\right)>0$. Thus, if $\theta_{m}=1$, there is a root of the polynomial in the interval $\left(x_{2 m}, x_{2 m+1}\right)$. Hence the number of roots in the interval $\left(x_{2 m_{0}}, x_{2 k+1}\right)$ must exceed $\sum_{m=m_{0}}^{k} \theta_{m}$ where $m_{0}=[k / 2]+1$.

2.5. We have

$$
\left|\sum_{m=m_{0}}^{k}\left\{\theta_{m}-E\left(\eta_{m}\right)\right\}\right| \leq\left|\sum_{m=m_{0}}^{k}\left\{\eta_{m}-E\left(\eta_{m}\right)\right\}\right|+\sum_{m=m_{0}}^{k} \rho_{m} .
$$

Let $A(w)$ be the set of $w$ for which

$$
\sup _{k-m_{0}+1 \geq k_{0}} \frac{1}{k-m_{0}+1}\left|\sum_{m=m_{0}}^{k}\left\{\theta_{m}-E\left(\eta_{m}\right)\right\}\right|>\varepsilon,
$$

$B(w)$ be the set of $w$ for which

$$
\sup _{k-m_{0}+1 \geq k_{0}} \frac{1}{k-m_{0}+1}\left|\sum_{m=m_{0}}^{k}\left\{\eta_{m}-E\left(\eta_{m}\right)\right\}\right|>\varepsilon / 2
$$

and $C(w)$ be the set of $w$ for which

$$
\sup _{k-m_{0}+1 \geq k_{0}} \frac{1}{k-m_{0}+1} \sum_{m=m_{0}}^{k} \rho_{m}>\varepsilon / 2 .
$$

Since $E\left(\rho_{m}\right)=1, P\left(\rho_{m}=1\right)$,

$$
\begin{aligned}
E\left(\rho_{m}\right) & =P\left\{\left(\left|R_{2 m}\right| \geq V_{2 m}\right) \cup\left(\left|R_{2 m+1}\right| \geq V_{2 m+1}\right)\right\} \\
& \leq P\left(\left|R_{2 m}\right| \geq V_{2 m}\right)+P\left(\left|R_{2 m+1}\right| \geq V_{2 m+1}\right) .
\end{aligned}
$$

Using Lemma 3 and (2.3) we have $E\left(\rho_{m}\right)<\mu / m^{2-\varepsilon}$. Therefore

$$
\frac{1}{k-m_{0}+1} \sum_{m=m_{0}}^{k} E\left(\rho_{m}\right) \leq \frac{1}{k-m_{0}+1} \sum_{m=m_{0}}^{k}\left(\mu / m^{2-\varepsilon}\right)<\mu / / m_{0}^{2-\varepsilon}
$$

and so

$$
\begin{aligned}
P\{C(w)\} & <\sum_{k-m_{0}+1 \geq k_{c}} P\left\{\frac{1}{k-m_{0}+1} \sum_{m=m_{0}}^{k} \rho_{m}>\varepsilon / 2\right\} \\
& <\left(2 \mu^{\prime} / \varepsilon\right) \sum_{k-m_{0}+1 \geq k_{0}}\left(1 / m_{0}^{2-\varepsilon}\right) .
\end{aligned}
$$


Here we need the strong law of large numbers in following form, which is a consequence of the Hajek-Renyi inequality (see [1]):

LEMMA 4. Let $\eta_{1}, \eta_{2}, \ldots$ be a sequence of independent random variables with $V\left(\eta_{i}\right)<1$ for all $i$. Then, for each $\varepsilon>0$,

$$
\operatorname{Pr}\left\{\sup _{k \geq k_{0}}\left|\frac{1}{k} \sum_{i=1}^{k}\left\{\eta_{i}-E\left(\eta_{i}\right)\right\}\right| \geq \varepsilon\right\} \leq \frac{D}{\varepsilon^{2} k_{0}},
$$

where $D$ is a positive constant.

Applying Lemma 4, we have

$$
P\{B(w)\}<4 D /\left(\varepsilon^{2} k_{0}\right)=\mu_{3} / k_{0} .
$$

From (2.19) it follows that $A(w) \subseteq B(w) \cup C(w)$. Therefore

$$
P\{A(w)\}<\mu_{3} / k_{0}+\mu_{4} \sum_{k-m_{0}+1 \geq k_{0}}\left(1 / m_{0}^{2-\varepsilon}\right) .
$$

Hence

$$
\sup _{k-m_{0}+1 \geq k_{0}} \frac{1}{k-m_{0}+1}\left|\sum_{m=m_{0}}^{k}\left\{\theta_{m}-E\left(\eta_{m}\right)\right\}\right|<\varepsilon
$$

outside the set $A(w)$ where $P\{A(w)\}<\mu_{3} / k_{0}+\mu_{4} \sum_{k-m_{0}+1 \geq k_{0}}\left(1 / m_{0}^{2-\varepsilon}\right)$. Therefore

$$
\frac{1}{k-m_{0}+1} \sum_{m=m_{0}}^{k} \theta_{m}>\frac{1}{k-m_{0}+1} \sum_{m=m_{0}}^{k} E\left(\eta_{m}\right)-\varepsilon
$$

for all $k$ such that $k-m_{0}+1 \geq k_{0}$. So that

$$
\begin{aligned}
N_{n}(w) & >\sum_{m=m_{0}}^{k} \theta_{m}>\left(k-m_{0}+1\right)(\delta-\varepsilon) \\
& =(k-[k / 2])(\delta-\varepsilon)>k(\delta-\varepsilon) / 2>\frac{\mu_{1}(\delta-\varepsilon)}{2 \sqrt{c_{1}}} \varepsilon_{n} \log n,
\end{aligned}
$$

for all $k$ such that $k-m_{0}+1 \geq k_{0}$, that is, for all $n>n_{0}$. We have

$$
\begin{aligned}
P\{A(w)\} & <\mu_{3} / k_{0}+\mu_{4} \sum_{k \geq 2 k_{0}-1}\left(1 / m_{0}^{2-\varepsilon}\right)<\mu_{3} / k_{0}+\mu_{4} \sum_{k \geq k_{0}}\left(1 / k^{2-\varepsilon}\right) \\
& <\mu / k_{0}<\left(\frac{\mu \sqrt{C_{1}}}{\mu_{1}}\right) /\left(\varepsilon_{n_{0}} \log n_{0}\right) .
\end{aligned}
$$

Now the result follows by taking $C_{1}=\mu_{1}^{2}(\delta-\varepsilon)^{2} / 4$. 


\section{References}

[1] J. Hajek and A. Renyi, 'A generalisation of an inequality of Kolmogorov', Acta Math. Hungar. 6 (1955), 281-283.

[2] I. A. Ibragimov and N. B. Maslova, 'On the expected number of real zeros of random algebraic polynomials I. Coefficients with zero means' (translated by B. Seckler), Theory Probab. Appl. 16 (1971), 228-248.

[3] I. A. Ibragimov and Yu. V. Linnik, Independent and stationary sequences of random variables (Wolters-Noordhoff, Groningen, 1972).

[4] M. N. Mishra, N. N. Nayak and S. Pattanayak, 'Lower bound of the number of real roots of a random algebraic polynomial', J. Indian Math. Soc. 45 (1981), 285-296.

[5] ___, 'Strong result for real zeros of random polynomials', Pac. J. Math. 103 (1982), 509-522.

[6] _ _ 'Lower bound for the number of real roots of a random algebraic polynomial', $J$. Austral. Math. Soc. Series A 35 (1983), 18-27.

[7] G. Samal, 'On the number of real roots of a random algebraic equation', Proc. Camb. Philos. Soc. 58 (1962) 433-442.

[8] _ , and D. Pratihari, 'Strong result for real zeros of random polynomials', J. Indian Math. Soc. 40 (1976), 223-234.

[9] _ , 'Strong result for real zeros of random polynomials II', J. Indian Math. Soc. 41 (1977), 395-403.

[10] _ 'Number of real zeros of a random algebraic polynomial', Indian J. Math. 20 (3) (1978), 225-232.

\section{College of Basic Sciences \& Humanities}

Bhubaneswar - 751 003, Orissa

INDIA 